ORIGINAL ARTICLE

\title{
SAFETY AND THERAPEUTIC EFFECT OF METRONOMIC CHEMOTHERAPY WITH CYCLOPHOSPHAMIDE AND CELECOXIB IN ADVANCED BREAST CANCER PATIENTS
}

1*Herman A. Perroud, ${ }^{1 *}$ Maria José. Rico, ${ }^{2}$ Carlos M. Alasino, ${ }^{2}$ Francisco Queralt, ${ }^{3}$ Stella M. Pezzotto, ${ }^{1}$ Viviana R. Rozados, ${ }^{1}$ O. Graciela Scharovsky.

${ }^{1}$ Institute of Experimental Genetics, School of Medical Sciences, National University of Rosario, Argentina; ${ }^{2}$ Rosario Institute of Oncology, Rosario, Argentina; ${ }^{3}$ Institute of Immunology, School of Medical Sciences, National University of Rosario, Argentina; *Contributed equally

\section{Corresponding author:}

Dr. O. Graciela Scharovsky

Santa Fe 3100

(S2002KTR) Rosario, Argentina

TE: + 54-341-4804558/63 Ext: 244

Fax: + 54-341-4804569

e-mail: graciela.scharovsky@gmail.com 


\begin{abstract}
Metronomic chemotherapy (MCT), the chronic administration, at regular intervals, of low doses of chemotherapeutic drugs, without extended rest periods, allows chronic treatment with therapeutic efficacy and low toxicity. Our pre-clinical results suggested that combined MCT with cyclophosphamide (CY) and celecoxib (CEL) could inhibit breast cancer growth. The aim of this study was to determine the toxicity, safety and efficacy of the oral chronic administration of CY $50 \mathrm{mg}$ p.o. daily, plus CEL $400 \mathrm{mg}$ (200 mg p.o. bid) in advanced breast cancer patients (ABCP). The therapeutic response consisted in stable disease in a high proportion of patients and one partial response. The overall clinical benefit rate obtained was $66.7 \%$. Toxicity was low. Evaluation of quality of life showed no changes during the response period. MCT with CY $+\mathrm{Cel}$ is safe and shows therapeutic effect in ABCP.
\end{abstract}

\title{
KEYWORDS
}

Breast cancer - metronomic chemotherapy - cyclophosphamide - celecoxib Biomarkers

Argentine Regulatory Agency Number: 4596/2009. 


\section{INTRODUCTION}

Breast cancer is the most common cancer and is the second leading cause of cancer death in American women, exceeded only by lung cancer [1]. In Argentine during 2008, there were 104.859 new cases of malignant tumors, being breast cancer the malignant illness with higher incidence in women (74/100000 women) [2].

Conventional cancer therapies (surgery, radiotherapy, chemotherapy) seem to have reached an efficacy therapeutic plateau with relative toxicity and detriment in the quality of life. Those characteristics accounts for increased costs in public health due to the need of implementing compensatory treatments and hospital admissions.

A turning point in cancer chemotherapy can be placed in the year 2000, when Fidler and Ellis stated, "Cancer is a chronic disease and should be treated like other chronic diseases" [3]. In agreement with this concept, several groups, including ours, began to study a new modality of drug administration that Douglas Hanahan named "metronomic therapy" [4]. It consists of the administration of chronic, equally spaced and, generally, low doses of chemotherapeutic drugs without extended rest periods, that allows chronic treatment with therapeutic efficacy and low toxicity.

Browder et al demonstrated the antiangiogenic effect of $\mathrm{Cy}$ using a low dose schedule; they also found that this effect was lost when using high doses and standard schedules due to the re-growth of tumor vasculature [5]. At variance with what it is observed when using standard chemotherapy schemes, they showed that metronomic chemotherapy (MCT) avoided drug resistance, a fact that may be attributed to the high genetic stability of the endothelial cell, the main target of MCT, less prone to develop drug resistance [6]. Similar results were also found by Klement et al [7]. Nevertheless, in a pre-clinical study, it was demonstrated resistance to MCT, although due to different mechanisms than those involved in resistance to drugs administered in the maximum tolerated dose (MTD) [8].

Tumor angiogenesis is a tumor micro-environmental process that promotes tumor cell survival and growth, invasion and metastasis [9]. Hence, antiangiogenic therapies would serve as antitumor ones. 
Several mechanisms account for the anti-angiogenic effect of MCT observed in vivo. These include, selective inhibition of proliferation and/or induction of apoptosis of activated endothelial cells, selective inhibition of endothelial cell migration, increase in the expression level of the endogenous angiogenesis inhibitor thrombospondin-1 (TSP1) and sustained decrease in levels and viability of bone marrow-derived endothelial progenitor cells [10]. However, the inhibition of angiogenesis would not be the exclusive mechanism of action of MCT since certain drugs, like $\mathrm{Cy}$, might contribute to the efficacy of the treatment through the stimulation of the immune response [10-12]

Cyclophosphamide (Cy), an alkylating drug that exerts a toxic effect on proliferating cells, is one of the most used drugs in cancer chemotherapy being one of the earliest cytotoxic drugs used in MCT [5].

Cyclooxygenase $2(\mathrm{COX}-2)$ is a prostaglandin synthase enzyme that has been implicated in multiple stages throughout the tumorigenic process [13], and that is overexpressed in a variety of malignancies including breast cancer, at variance with COX-1, which is constitutively expressed in normal tissues. Recently, elevated COX-2 expression in breast cancer has been also associated with the presence of distant metastases [14]. Celecoxib (Cel) is a selective inhibitor of COX-2 that has shown antiangiogenic [15] and antiproliferative [16] properties in breast cancer cell lines.

The antitumor and antimetastatic properties of MCT with Cy as a mono-therapy [17], or in combination with $\mathrm{Cel}$ [18], were demonstrated in our pre-clinical tumor-models. The promising results obtained, in terms of therapeutic efficacy, good tolerability and low toxicity profile together with previous clinical experience by others setting the metronomic dose of Cy [19, 20] prompted us to conduct a phase I/II trial employing metronomic administration of $\mathrm{Cy}$ combined with $\mathrm{Cel}$ for the treatment of metastatic breast cancer (MBC) patients progressing after standard chemotherapy.

In addition, we evaluated several biomarkers of angiogenesis including serum concentration of VEGF and TSP-1, and circulating (CECs) and progenitor (CEPs) endothelial cells, in an attempt to define their ability to predict response to the therapy. 


\section{PATIENTS AND METHODS}

\section{Eligibility}

Recruited patients were required to have histologically confirmed advanced breast cancer progressing after 3 , and no more than 4 , chemotherapy schemes. Other inclusion criteria were: age between 18 and 80 years; adequate bone marrow, renal and liver function, normal calcemia, at least one lesion according to RECIST criteria and more than 3 months of life expectancy. It was mandatory that all patients must be recovered from any prior chemotherapy, radiotherapy, or surgery before their inclusion. Each patient included in this study gave her written informed consent. This study was authorized by the School of Medicine Bioethics Committee and by A.N.M.A.T. (Argentine Regulatory Agency).

\section{Treatment and Evaluation}

All patients received Cy 50 mg p.o. daily, plus Cel 400 mg (200 mg p.o. bid).

Baseline evaluation included clinical examination, chest X-ray, liver ultrasound or CT scan, and complete biochemical and hematologic tests. Patients were evaluated by physical examination and hematologic tests every 14 days during the first 6 months and every 28 days thereafter.

Clinical response and toxicity were evaluated every two months or earlier if it was necessary. Patients were followed until progression or death.

In case of $\mathrm{G}$ (grade) II neutropenia or thrombocytopenia, the dose of $\mathrm{Cy}$ was reduced to $50 \%$ ( $25 \mathrm{mg} /$ day), while if reaching GIII toxicity it was reduced to $25 \%$ of original dose (12.5 mg/day). Treatment was discontinued until hematologic recovery in case of GIV toxicity.

In case of nausea and vomiting, dyspepsia, or GIII abdominal pain, Cel was reduced to $50 \%$ (200 mg/day). More than two dose reductions or any gastrointestinal bleed GII or more resulted in study discontinuation. 


\section{Evaluation of CECs and CEPs}

Quantification of CECs and CEPs by flow cytometry was carried out on peripheral blood collected in tubes with EDTA and then separated by Ficoll-Hypaque gradient. Samples were collected at baseline, at the first three visits, and every 2 months thereafter. A panel of monoclonal antibodies, including anti-CD45-FITC (BD Pharmingen,USA) to exclude hematopoietic cells, anti-CD31-ALEXA Fluor ${ }^{\circledR} 488$ (BD Pharmingen) and anti-CD133-PE (Miltenyi Biotec, Germany) were used to determine the percentages of CECs and CEPs, respectively. Samples were evaluated using a Coulter Epics XL Flow cytometer.

\section{VEGF and TSP-1 quantification}

Levels of VEGF and TSP-1 were evaluated by ELISA in serum samples collected at baseline, at the first three visits, and every 2 months thereafter, with VEGF Quantikine (R\&D Systems) and TSP-1 Quantikine (R\&D Systems) kits. Blood samples were allowed to clot for $2 \mathrm{~h}$ at room temperature. After centrifugation, the serum was removed and stored at $-20^{\circ} \mathrm{C}$ until used. The ELISA method was performed in duplicates, according to the manufacturer instructions.

\section{Adverse events and quality of life}

Toxicity was evaluated according to the CTCAE (National Cancer Institute's Common Terminology Criteria for Adverse Events) version 3.0. Life quality was evaluated by ECOG scale (Eastern Cooperative Oncologic Group) and FACT-B (The Functional Assessment of Cancer Therapy Breast) questionnaires. Physical well-being (GP), Social/family well-being (GS), Functional well-being (GF), Emotional well-being (GE), and Additional concerns (AC) were analyzed at three time points: baseline (BL); middle (MT) and end of treatment (ET).

\section{Study End Points}

The primary end point was the overall clinical benefit (OCB), which was defined as complete response (CR), partial response (PR), or stable disease (SD) $\geq 8$ weeks. Tumor assessments were performed every 2 months according to WHO standard criteria and response was evaluated by Response Evaluation Criteria in Solid Tumors (RECIST). Safety and tolerability were determined on the basis of the presence and grade of 
adverse events. Time to progression (TTP) was calculated from baseline to the first documentation of progressive disease (PD) or related death if this occurred before PD. Patients with death unrelated to breast cancer or this treatment were censored in TTP analysis. Response Duration (RD) was calculated from date of first documented response (ie, CR, PR or SD) until date of first documented PD.

\section{Study design and statistical analysis}

For this nonrandomized phase I/II trial, sample size calculation was based on an optimal two-stage minimax design [21]. This approach was used to investigate whether the overall response rate is sufficient to proceed to phase III trials with a minimum number of patients. The sample size for the trial was determined as follows. The threshold response rate was defined at $5 \%$ and the expected response rate was set at $25 \%$. This study was regarded to be adequate to recruit a total of 25 patients (15 in the first stage and an additional 10 in the second), assuming an alpha error of 0.05 and beta error of 0.10 based on Simon two-stage Phase II design [22]. The decision to continue or not to the second stage is based on the response rate obtained in the first one. If any response is obtained in at least one patient, the second stage will be carried out.

The results were statistically analyzed, depending on the variable distribution, using parametric or non-parametric tests. Friedman and Wilcoxon signed-rank tests were used to assess whether median scores of FACT-B items were significantly different from baseline. Differences in VEGF and TSP-1 levels were evaluated by linear regression test. Kaplan Meier analysis was used to evaluate duration of response. Data were analyzed using the STATA statistical software (version 6) [23]. 


\section{RESULTS}

\section{Patient characteristics}

Fifteen patients were enrolled, thus concluding the first stage of the study, while the enrollment of patients continues until completion of the pre-established number of 25 . Baseline characteristics of patients and tumors are depicted in Table 1. The median age was 61 years (range: 38-78). All patients were heavily pretreated, 7/15 and 8/15 received 3 and 4 prior chemotherapy regimens, respectively. The most frequent metastases locations were liver and bone.

\section{Toxicity and adverse events}

The hematologic adverse events associated with the therapy were: Anemia G2 ( $n=4 / 15)$, leukopenia G1 $(n=2 / 15)$ and G2 $(n=2 / 15)$, neutropenia G1 $(n=1 / 15)$ and G2 $(n=2 / 15)$, thrombocytopenia GI $(n=1 / 15)$. Those events were easily overcome with transitory reduction of Cy doses (Online Resource 1). Four patients over fifteen developed G1 dyspepsia related to the treatment, a toxicity that did not affect compliance and reversed easily with the administration of omeprazole $20 \mathrm{mg}$ v.o. daily. All the adverse events, whether related or not to the therapy are summarized in Table 2.

There was no evidence of hepatic, renal or cardiac toxicities associated with the therapy. Minimal changes in liver enzymes (lactate dehydrogenase and alkaline phosphatase) were likely due to the underlying disease or related to disease progression. $\mathrm{Ca}$ 15-3 concentrations increased at the time of disease progression, and those values, along with CT scan, were useful to identify patients who were unresponsive to therapy (Online Resource 2).

\section{Therapeutic response}

A partial response (PR) was observed in 1 patient (6.7\%), which lasted 6 weeks. Stable disease (SD) was observed in 9/15 patients (60\%). Median RD among patients with stable disease was 19.24 weeks (range: 4-73.6). The OCB obtained was $66.7 \%$ $(\mathrm{PR}=1 / 15+\mathrm{SD}=9 / 15)$. The median TTP was 14 weeks (range: $3.7-81.6)$ for responder patients. The evolution of the percentage of progression free survival is shown in Fig.1.

\section{Biomarkers}

The percentages of CECs and CEPs were assessed along the treatment a non-clear trend variation was observed in the percentages of CECs and CEPs. The same behavior was 
obtained when patients were classified in responder $(\mathrm{R})$ and non-responder $(\mathrm{NR})$ for the percentages of CECs in R (Fig.2A), NR (Fig.2B) and of CEPs in R (Fig. 2C). Interestingly, NR patients showed a tendency to increase the values of CEPs (Fig. 2D) during their permanence in the study.

Serum concentration of VEGF decreased as a function of time ( $\mathrm{P}=0.004)$ (Fig. 3A). On the other hand, TSP-1 levels did not show significant modifications (Fig. 3B), even when they were classified in R and NR.

\section{Life quality}

\section{FACT-B questionnaire}

During follow-up, when comparing the FACT-B items during response (from BL to MT), no significant differences were observed. When questionnaires corresponding to the end of treatment (ET) were included in the statistical analysis, the item AC showed a significant increase $(\mathrm{P}=0.008)$, while for $\mathrm{GF}$ the increase was marginally significant $(\mathrm{P}=0.055)$ (Online resource 3$)$.

\section{ECOG evaluation}

In assessing the performance status by means of the ECOG scale, the value corresponding to the time of progression was not included. Four patients over 15 (26.7\%) were not evaluated since their permanence in the treatment was $\leq 8$ weeks. The analysis showed no changes in the score in $1 / 11(9 \%)$ patients, a worsened score in $4 / 11$ (36.4\%) patients, a transitory and a permanent improved score in 2/11 (18.2\%) and 4/11 (36.4\%) patients, respectively (Online Resource 4). 


\section{DISCUSSION}

Effective, noninvasive and low toxicity treatments for breast cancer represent a challenge for medical oncologists. For decades the pharmaceutical industry has been focused in developing antineoplastic agents with high cytotoxic effect on tumor cells. This effect is related to agent doses and is the main cause of anti-tumor efficacy; however it is also accompanied by toxicity and deterioration of quality of life.

Angiogenesis represents a key process in carcinogenesis. Antiangiogenic drugs interfere in many common pathways shared in all neoplastic cells in an unspecific fashion [24]. The mechanism of action of MCT proposed by Folkman and Kerbel points to the tumor endothelial cells which, indirectly, destroy naive and resistant cancer cells by induction of hypoxia and nutrients deprivation. These mechanisms had been validated in preclinical and clinical models. However, additional mechanisms of action may be involved like induction of tumor dormancy and immunomodulation [10, 12].

Based in our pre-clinical studies [18], we designed this trial to evaluate the safety, efficacy of MCT with Cy plus Cel in advanced breast cancer refractory to standard chemotherapy, along with the identification of possible biomarkers to predict response to therapy.

The OCB obtained was $66.7 \%$ ( $\mathrm{PR}=1 / 15$ and $\mathrm{SD}=9 / 15)$. This result is very encouraging, considering the stage of the disease and the low possibility of obtaining a long term clinical benefit after 3 or 4 lines of chemotherapy. Interestingly, we obtained similar results to those achieved with standard regimens administered to metastatic breast cancer patients but avoiding toxicity. Accordingly, the use of gemcitabine and carboplatin plus iniparib yielded a 56\% OCB rate [25], while the administration of capecitabine and vinorelbine in HER2-negative patients reached a OCB rate of $70 \%$ [26] and the combination of paclitaxel with gemcitabine resulted in a $80 \%$ OCB rate [27].

In agreement to our findings, other promising results using MCT were obtained in patients with metastatic breast cancer treated with Cy plus Methotrexate (Mtx) [28], Cy plus Mtx and Thalidomide [29], Cy plus Mtx and Trastuzumab in Her2/neu+ patients [30]; besides, it was recently demonstrated that MCT with capecitabine displayed good 
therapeutic activity in advanced breast cancer [31]. Also, in patients with colorectal cancer at high risk of recurrence, adjuvant postoperative MCT using Irinotecan plus Uracil and Tegafur improved overall survival rates [32]. Moreover, patients with advanced hormone refractory prostate cancer treated with a single standard dose of $\mathrm{Cy}$ followed by MCT with Cel and Dexamethasone showed biological activity and low toxicity profile [33]. On the other hand, OA Khan et al, in a large Phase II trial including heavily pretreated patients with different types of cancer (breast, gastrointestinal, melanoma, ovarian, prostate, renal) and treated with daily $\mathrm{Cy}, \mathrm{Cel}$ and weekly Mtx found no toxicity, but also non-objective response, and short duration of SD [34].

We documented long term SD in $60 \%$ of the patients who showed median TTP and RD of 14 and 19.2 weeks, respectively. These results are similar to those found by Salem et al. [35], with a MRD of $4 \pm 0.84$ months, and lower than the 6.8 months found by Colleoni et al. [28].

We found very low toxicity, being myelosuppression the most common event associated to $\mathrm{Cy}$ treatment. Interestingly, it was easily reverted by temporary dose reduction. Gastrointestinal toxicity was also minimal. Compared to other standard schemes of treatment [36], the side effects derived from the present treatment were minimal.

Several authors have demonstrated, in pre-clinical models, the therapeutic effect of metronomic administration of $\mathrm{Cy}$ in combination with various antiangiogenic agents such as angiogenesis inhibitor TNP-470 [5], imatinib [37], the angiogenic peptide ABT510 [38], tirapazamine [39], cetuximab, anti-EGFR [40], 5-fluorouracil prodrug UFT [41], the angiogenesis inhibitor axitinib [42] and others, in the treatment of different types of tumors, including lung cancer and leukemia [5], pancreatic carcinoma [37], prostate [38], colon [39], and breast [41].

One of the most important angiogenic molecules is VEGF, which stimulates adhesion, proliferation and survival of normal endothelial cells. While its expression is strictly regulated in normal tissue, the overexpression of VEGF has been observed in several tumor models, associated to abnormal blood vessels [6]. On the other hand, antiangiogenic factors like TSP-1 inhibiting proliferation, growth, motility and adhesion of tumor endothelial cells may be involved in the regulation of tumor angiogenesis [43]. 
Also, the recruitment of hematopoietic cells and circulating endothelial progenitor cells (CEP) derived from bone marrow, are necessary for tumor angiogenesis [44]. The CEPs, which can be mobilized from the bone marrow by growth factors such as VEGF, cytokines or ischemia [45], would be a MCT cell target, as well [46].

In order to find possible markers of response to therapy, we evaluated serum levels of VEGF and TSP-1 and percentages of circulating CECs and CEPs. Serum VEGF showed a significant decrease over time, supporting the anti-angiogenic effect of the treatment. However, the limitations in the number of patients included so far preclude from assuring its usefulness as a marker of response. Different results were obtained in a Phase II study conducted with bevacizumab and metronomic oral dose of $\mathrm{Cy}$ in patients with recurrent ovarian tumors, in which no correlation was found between VEGF levels and response to treatment [47]. On the other hand, treatment with MCT with daily Cy, dalteparin and prednisone and twice-weekly dose of Mtx in patients with metastatic breast cancer, showed clinical activity, a non-significant decrease of VEGF, along with a significant increase in receptors sVEGFR-1 and sVEGFR-2 after two weeks of therapy [48], although a combination of $\mathrm{Cy}$, Cel and Mtx showed negligible activity in advanced cancer and the analysis of several biomarkers (included VEGF) indicated minimal effects on endothelium [34].

Thrombospondin 1 is a potent and highly specific inhibitor of angiogenesis and it is considered a secondary mediator of the antiangiogenic effect of some MCT regimens [49]. However, MCT with Cy + Cel did not significantly modify, in our patients, TSP-1 serum concentration. Moreover, those values did not correlate to disease progression or treatment response. Accordingly, several studies also failed to find such a correlation. In a trial of MCT with $\mathrm{Cy}+\mathrm{Cel}$ and Dexamethasone in advanced hormone-refractory prostate cancer patients [33], the plasma TSP-1 level, while highly variable, increased during treatment in both, responsive and unresponsive patients. In another trial comparing metronomic versus MTD of cisplatin and docetaxel given i.v. in patients with lung cancer, no significant changes in VEGF, TSP1 and VEGFR1 levels were found in the group treated with MCT [50]. Recently it was shown a decrease in TSP-1 levels in patients receiving metronomic $\mathrm{Cy}$ a change that did not correlate to clinical benefit [51] 
The proportion of CEPs in NR patients showed a tendency to higher values during their time of permanence. Accordingly, a study in ovarian cancer patients showed that high circulating CEPs correlated with poor overall survival [52]. On the contrary, other studies in Hodgkin lymphoma [20] and breast cancer [19] patients, showed a decrease of CEPs and/or CECs, when treated with Cy plus Cel or Cy plus capecitabine and bevacizumab, respectively Our preliminary results, together with data from other reports, are yet scarce to consider the use of CEPs or CECs as biomarkers to identify patients who are candidates to antiangiogenic therapy, but may be useful to evaluate treatment response after its initiation.

One common outcome when employing MTD regimens is a deterioration of life quality. On the contrary, what we have found so far is that quality of life, assessed by the FACT-B questionnaire, showed no changes during the response period. This is an interesting finding, considering the type of patients we are dealing with. When the questionnaire corresponding to the end of treatment was also included for the statistical analysis, the only item that showed a significant increase was AC. The increase of the additional concerns could be likely due to fear, frustration or to the knowledge of lack of additional curative treatments. Moreover, assessment of functional status using the ECOG scale showed that $70 \%$ of patients improved or did not change their condition. Such a result may be mainly attributed to stable disease, noninvasive drug administration, low toxicity of the treatment, anti-inflammatory effect of Cel and also, to a subjective factor from the patient.

In conclusion, $\mathrm{MCT}$ combining $\mathrm{Cy}$ and $\mathrm{Cel}$ for treating advanced breast cancer showed very low toxicity and therapeutic response in a high proportion of patients. The decrease of serum VEGF confirmed the antiangiogenic nature of the drug combination and therapeutic schedule. There was no deterioration of the life quality in a high proportion of patients, a result that represents one of the most important rewards, considering their stage of disease progression at the beginning of the therapy. 


\section{FUTURE PERSPECTIVE}

The promising results obtained in the first stage of the trial warrants not only its prosecution to the second stage but also the design of new protocols targeting to patients in earlier stages of the disease, on its completion.

\section{EXECUTIVE SUMMARY}

Metronomic chemotherapy with cyclophosphamide plus celecoxib administered to advanced breast cancer patients yielded the following results:

- The adverse events related to the therapy where few and mild (GI or GII), mainly hematologic and gastrointestinal.

- The OCB obtained was $66.7 \%(\mathrm{PR}=1 / 15+\mathrm{SD}=9 / 15)$. The median TTP and RD was 14 and 19.2 weeks.

- Serum concentration of VEGF decreased significantly as a function of time.

- CEPs showed a tendency to higher values during treatment in non-responder patients

- When comparing the FACT-B items during response no significant differences were observed.

- The performance status measured by the ECOG scale showed no modification or improvement in $7 / 11(63.6 \%)$ of the responder patients. 


\section{ACKNOWLEDGMENTS}

We would like to thank Cibic S.A. and especially to Dr. Ricardo Giordano, for their help in flow cytometry studies.

This work was supported by ANPCyT (Agencia Nacional de Promoción Científica y Tecnológica) grant (PICT 2006/1908 to OGS, VRR y SMP). HAP is fellow of ANPCyT. MJR is fellow of CONICET (Consejo Nacional de Investigaciones Científicas y Técnicas).

\section{ETHICAL STANDARDS}

The authors declare that the protocol herein described comply with the current laws of Argentina. The protocol was authorized by the School of Medicine Bioethics Committee and by A.N.M.A.T. (Argentine Regulatory Agency).

\section{CONFLICT OF INTEREST}

The authors declare that they have no conflict of interest. 


\section{REFERENCES}

1. American Cancer Society: Breast Cancer Detailed Guide. (18 september 2011), (2010).

2. Instituto Nacional Del Cáncer: Información para Equipos de salud. Análisis de situación del cáncer en la Argentina. (19 March 2012), (2010).

3. Fidler IJ, Ellis LM: Chemotherapeutic drugs--more really is not better. Nat Med 6(5), 500-502 (2000).

4. Hanahan D, Bergers G, Bergsland E: Less is more, regularly: metronomic dosing of cytotoxic drugs can target tumor angiogenesis in mice. J Clin Invest 105(8), 1045-1047 (2000).

5. Browder $\mathrm{T}$, Butterfield $\mathrm{CE}$, Kraling $\mathrm{BM}$ et al.: Antiangiogenic scheduling of chemotherapy improves efficacy against experimental drug-resistant cancer. Cancer Res 60(7), 1878-1886 (2000).

6. Kerbel RS, Kamen BA: The anti-angiogenic basis of metronomic chemotherapy. Nat Rev Cancer 4(6), 423-436 (2004).

7. Klement G, Baruchel S, Rak J et al.: Continuous low-dose therapy with vinblastine and VEGF receptor-2 antibody induces sustained tumor regression without overt toxicity. J Clin Invest 105(8), R15-24 (2000).

8. Emmenegger $U$, Francia $G$, Chow $A$ et al.: Tumors that acquire resistance to low-dose metronomic cyclophosphamide retain sensitivity to maximum tolerated dose cyclophosphamide. Neoplasia 13(1), 40-48 (2011).

9. Carmeliet P: Angiogenesis in life, disease and medicine. Nature 438(7070), 932-936 (2005).

10. Pasquier $\mathrm{E}$, Kavallaris $\mathrm{M}$, Andre $\mathrm{N}$ : Metronomic chemotherapy: new rationale for new directions. Nat Rev Clin Oncol 7(8), 455-465 (2010).

11. Ghiringhelli F, Menard C, Puig PE et al.: Metronomic cyclophosphamide regimen selectively depletes CD4+CD25+ regulatory $T$ cells and restores $T$ and NK effector functions in end stage cancer patients. Cancer immunology, immunotherapy : Cll 56(5), 641-648 (2007).

12. Rozados VR, Mainetti LE, Rico MJ, Zacarias Fluck MF, Matar P, Scharovsky OG: The immune response and the therapeutic effect of metronomic chemotherapy with cyclophosphamide. Oncol Res 18(11-12), 601-605 (2010).

13. Gately S, Kerbel R: Therapeutic potential of selective cyclooxygenase-2 inhibitors in the management of tumor angiogenesis. Prog Exp Tumor Res 37, 179-192 (2003).

14. Fabi A, Metro G, Papaldo P et al.: Impact of celecoxib on capecitabine tolerability and activity in pretreated metastatic breast cancer: results of a phase II study with biomarker evaluation. Cancer Chemother Pharmacol 62(4), 717-725 (2008).

15. Farooqui M, Li Y, Rogers T et al.: COX-2 inhibitor celecoxib prevents chronic morphineinduced promotion of angiogenesis, tumour growth, metastasis and mortality, without compromising analgesia. Br J Cancer 97(11), 1523-1531 (2007).

16. Bocca C, Bozzo F, Bassignana A, Miglietta A: Antiproliferative effects of COX-2 inhibitor celecoxib on human breast cancer cell lines. Mol Cell Biochem 350(1-2), 59-70 (2011).

17. Rozados VR, Sanchez AM, Gervasoni SI, Berra HH, Matar P, Graciela Scharovsky O: Metronomic therapy with cyclophosphamide induces rat lymphoma and sarcoma regression, and is devoid of toxicity. Ann Oncol 15(10), 1543-1550 (2004).

18. Mainetti LE, Rozados VR, Rossa A, Bonfil RD, Scharovsky OG: Antitumoral and antimetastatic effects of metronomic chemotherapy with cyclophosphamide combined with celecoxib on murine mammary adenocarcinomas. I Cancer Res Clin Oncol 137(1), 151-163 (2011). 
19. Dellapasqua S, Bertolini F, Bagnardi $V$ et al.: Metronomic cyclophosphamide and capecitabine combined with bevacizumab in advanced breast cancer. Journal of clinical oncology : official journal of the American Society of Clinical Oncology 26(30), 48994905 (2008).

20. Buckstein R, Kerbel RS, Shaked $Y$ et al.: High-Dose celecoxib and metronomic "lowdose" cyclophosphamide is an effective and safe therapy in patients with relapsed and refractory aggressive histology non-Hodgkin's lymphoma. Clin Cancer Res 12(17), 51905198 (2006).

21. Simon R: Optimal two-stage designs for phase II clinical trials. Controlled clinical trials 10(1), 1-10 (1989).

22. Machin D, Campbell MJ, Tan SB, Tan SH: Phase II Trials. In: Sample Size Tables for Clinical Studies, (Ed.^(Eds). Wiley-Blackwell, 207-236 (2009).

23. Statacorp.: Stata Statistical Software: Release 6.0. College Station, Texas, USA (1999).

24. Torrisi R, Bagnardi V, Cardillo A et al:: Preoperative bevacizumab combined with letrozole and chemotherapy in locally advanced ER- and/or PgR-positive breast cancer: clinical and biological activity. Br J Cancer 99(10), 1564-1571 (2008).

25. O'shaughnessy J, Osborne C, Pippen JE et al.: Iniparib plus chemotherapy in metastatic triple-negative breast cancer. The New England journal of medicine 364(3), 205-214 (2011).

26. Gampenrieder SP, Bartsch R, Matzneller P et al.: Capecitabine and Vinorelbine as an All-Oral Chemotherapy in HER2-Negative Locally Advanced and Metastatic Breast Cancer. Breast Care (Basel) 5(3), 158-162 (2010).

27. Rau KM, Li SH, Chen SM et al.: Weekly paclitaxel combining with gemcitabine is an effective and safe treatment for advanced breast cancer patients. Jpn J Clin Oncol 41(4), 455-461 (2011).

28. Colleoni M, Rocca A, Sandri MT et al.: Low-dose oral methotrexate and cyclophosphamide in metastatic breast cancer: antitumor activity and correlation with vascular endothelial growth factor levels. Ann Oncol 13(1), 73-80 (2002).

29. Colleoni M, Orlando L, Sanna G et al:: Metronomic low-dose oral cyclophosphamide and methotrexate plus or minus thalidomide in metastatic breast cancer: antitumor activity and biological effects. Ann Oncol 17(2), 232-238 (2006).

30. Orlando L, Cardillo A, Ghisini R et al.: Trastuzumab in combination with metronomic cyclophosphamide and methotrexate in patients with HER-2 positive metastatic breast cancer. BMC cancer 6, 225 (2006).

31. Fedele $\mathrm{P}$, Marino A, Orlando L et al.: Efficacy and safety of low-dose metronomic chemotherapy with capecitabine in heavily pretreated patients with metastatic breast cancer. Eur J Cancer 48(1), 24-29 (2012).

32. Ogata $\mathrm{Y}$, Mori $\mathrm{S}$, Ishibashi $\mathrm{N}$ et al.: Metronomic chemotherapy using weekly lowdosage CPT-11 and UFT as postoperative adjuvant therapy in colorectal cancer at high risk to recurrence. J Exp Clin Cancer Res 26(4), 475-482 (2007).

33. Fontana A, Galli L, Fioravanti $A$ et al.: Clinical and pharmacodynamic evaluation of metronomic cyclophosphamide, celecoxib, and dexamethasone in advanced hormonerefractory prostate cancer. Clin Cancer Res 15(15), 4954-4962 (2009).

34. Khan OA, Blann AD, Payne MJ et al.: Continuous low-dose cyclophosphamide and methotrexate combined with celecoxib for patients with advanced cancer. $\mathrm{Br} J$ Cancer 104(12), 1822-1827 (2011).

35. Salem DA, Gado NM, Abdelaziz NN, Essa AE, Abdelhafeez ZM, Kamel TH: Phase II trial of metronomic chemotherapy as salvage therapy for patients with metastatic breast cancer. Journal of the Egyptian National Cancer Institute 20(2), 134-140 (2008). 
36. Cebon JS, Bishop JF, Harvey V, Mason B, Jeal PN: Dose-intense weekly cyclophosphamide, methotrexate, 5-fluorouracil, vincristine and prednisolone (CMFP) in advanced breast cancer. Br J Cancer 61(1), 133-136 (1990).

37. Pietras K, Hanahan D: A multitargeted, metronomic, and maximum-tolerated dose "chemo-switch" regimen is antiangiogenic, producing objective responses and survival benefit in a mouse model of cancer. Journal of clinical oncology : official journal of the American Society of Clinical Oncology 23(5), 939-952 (2005).

38. Yap R, Veliceasa D, Emmenegger U et al.: Metronomic low-dose chemotherapy boosts CD95-dependent antiangiogenic effect of the thrombospondin peptide ABT-510: a complementation antiangiogenic strategy. Clin Cancer Res 11(18), 6678-6685 (2005).

39. Emmenegger $\mathrm{U}$, Morton GC, Francia $\mathrm{G}$ et al:: Low-dose metronomic daily cyclophosphamide and weekly tirapazamine: a well-tolerated combination regimen with enhanced efficacy that exploits tumor hypoxia. Cancer Res 66(3), 1664-1674 (2006).

40. Du Manoir JM, Francia G, Man S et al.: Strategies for delaying or treating in vivo acquired resistance to trastuzumab in human breast cancer xenografts. Clin Cancer Res 12(3 Pt 1), 904-916 (2006).

41. Munoz R, Man S, Shaked $Y$ et al.: Highly efficacious nontoxic preclinical treatment for advanced metastatic breast cancer using combination oral UFT-cyclophosphamide metronomic chemotherapy. Cancer Res 66(7), 3386-3391 (2006).

42. Ma J, Waxman DJ: Modulation of the antitumor activity of metronomic cyclophosphamide by the angiogenesis inhibitor axitinib. Mol Cancer Ther 7(1), 79-89 (2008).

43. Taraboletti G, Rusnati M, Ragona L, Colombo G: Targeting tumor angiogenesis with TSP-1-based compounds: rational design of antiangiogenic mimetics of endogenous inhibitors. Oncotarget 1(7), 662-673 (2010).

44. Lyden $\mathrm{D}$, Hattori $\mathrm{K}$, Dias $\mathrm{S}$ et al.: Impaired recruitment of bone-marrow-derived endothelial and hematopoietic precursor cells blocks tumor angiogenesis and growth. Nat Med 7(11), 1194-1201 (2001).

45. Heeschen C, Aicher A, Lehmann R et al.: Erythropoietin is a potent physiologic stimulus for endothelial progenitor cell mobilization. Blood 102(4), 1340-1346 (2003).

46. Mancuso $\mathrm{P}$, Colleoni $\mathrm{M}$, Calleri $\mathrm{A}$ et al.: Circulating endothelial-cell kinetics and viability predict survival in breast cancer patients receiving metronomic chemotherapy. Blood 108(2), 452-459 (2006).

47. Garcia AA, Hirte H, Fleming $\mathrm{G}$ et al:: Phase II clinical trial of bevacizumab and low-dose metronomic oral cyclophosphamide in recurrent ovarian cancer: a trial of the California, Chicago, and Princess Margaret Hospital phase II consortia. Journal of clinical oncology : official journal of the American Society of Clinical Oncology 26(1), 7682 (2008).

48. Wong NS, Buckman RA, Clemons M et al.: Phase I/II trial of metronomic chemotherapy with daily dalteparin and cyclophosphamide, twice-weekly methotrexate, and daily prednisone as therapy for metastatic breast cancer using vascular endothelial growth factor and soluble vascular endothelial growth factor receptor levels as markers of response. Journal of clinical oncology : official journal of the American Society of Clinical Oncology 28(5), 723-730 (2010).

49. Bocci G, Francia G, Man S, Lawler J, Kerbel RS: Thrombospondin 1, a mediator of the antiangiogenic effects of low-dose metronomic chemotherapy. Proc Natl Acad Sci U S A 100(22), 12917-12922 (2003).

50. Tas F, Duranyildiz D, Soydinc HO et al.: Effect of maximum-tolerated doses and lowdose metronomic chemotherapy on serum vascular endothelial growth factor and 
thrombospondin-1 levels in patients with advanced nonsmall cell lung cancer. Cancer Chemother Pharmacol 61(5), 721-725 (2008).

51. Lansiaux A, Salingue S, Dewitte A, Clisant S, Penel N: Circulating thrombospondin 1 level as a surrogate marker in patients receiving cyclophosphamide-based metronomic chemotherapy. Investigational new drugs 30(1), 403-404 (2012).

52. Su Y, Zheng L, Wang $Q$ et al.: Quantity and clinical relevance of circulating endothelial progenitor cells in human ovarian cancer. J Exp Clin Cancer Res 29, 27 (2010). 


\section{Figure Legends}

Fig.1. Evolution of the percentage of progression free survival

Fig.2. Individual variation of percentage of CECs (A and B) and CEPs (C and D) in responder $(n=10)$ and non-responder $(n=5)$ patients, respectively.

Fig.3. Individual variations of VEGF (A) and TSP-1 (B) serum concentrations. The grey zones indicate the range of normal values. Linear regression analysis; A) $\mathrm{r}^{2}=0.113$; $\mathrm{P}=0.0042 ; \mathrm{B}) \mathrm{r}^{2}=0.002 ; \mathrm{P}=0.71$.

\section{On-Line Resources}

Online Resource 1. Evolution of hematologic parameters

Online Resource 2. Evolution of serologic parameters

Online Resource 3. FACT B questionnaire

Online Resource 4. Evolution of Performance status 
Figure 1

\section{Survival}

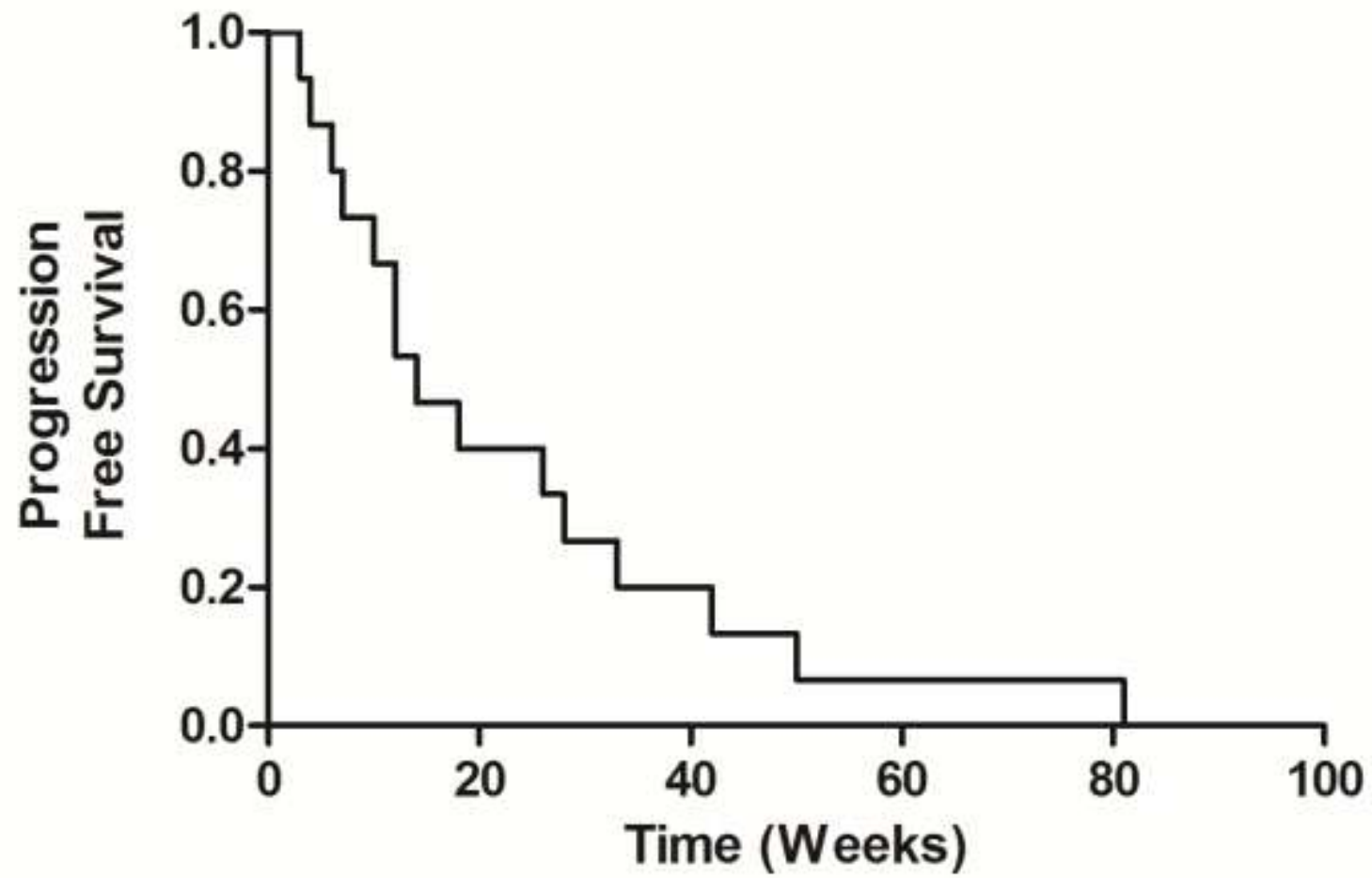

No. at risk 15

9

3

1

0 
Figure 2

\section{Circulating Endothelial Cells}

A)

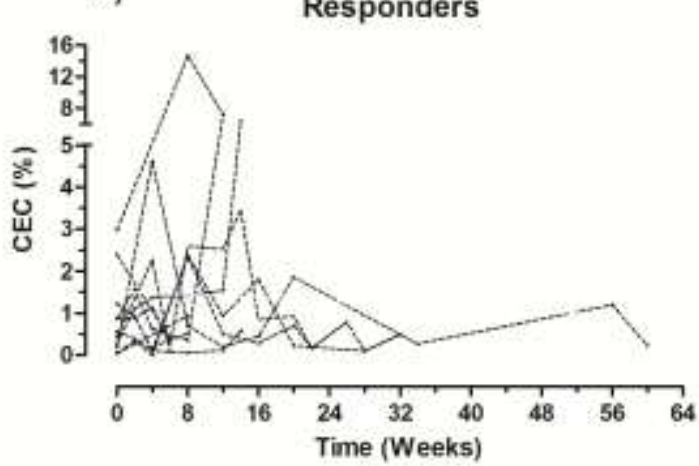

B)

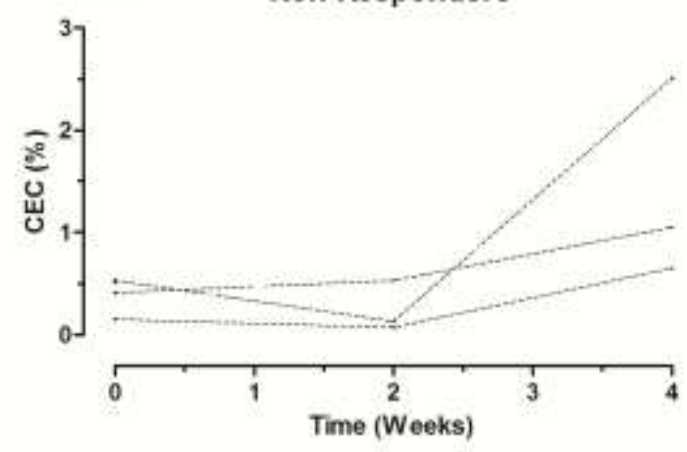

Progenitor Endothelial Cells

C)

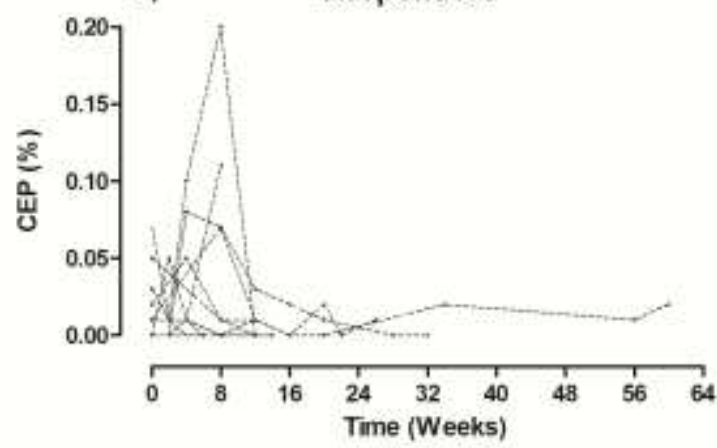

D)

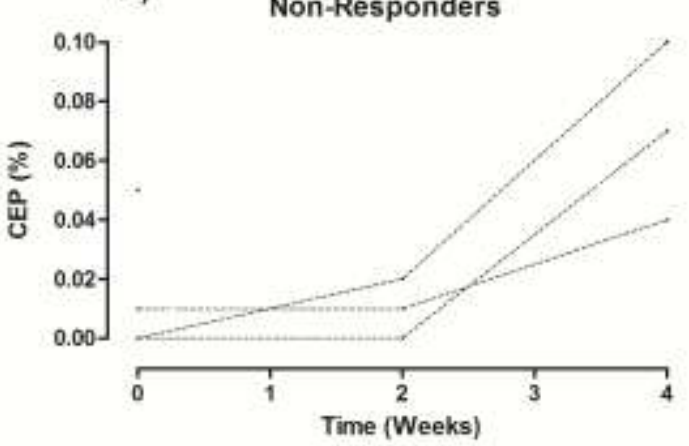


Figure 3
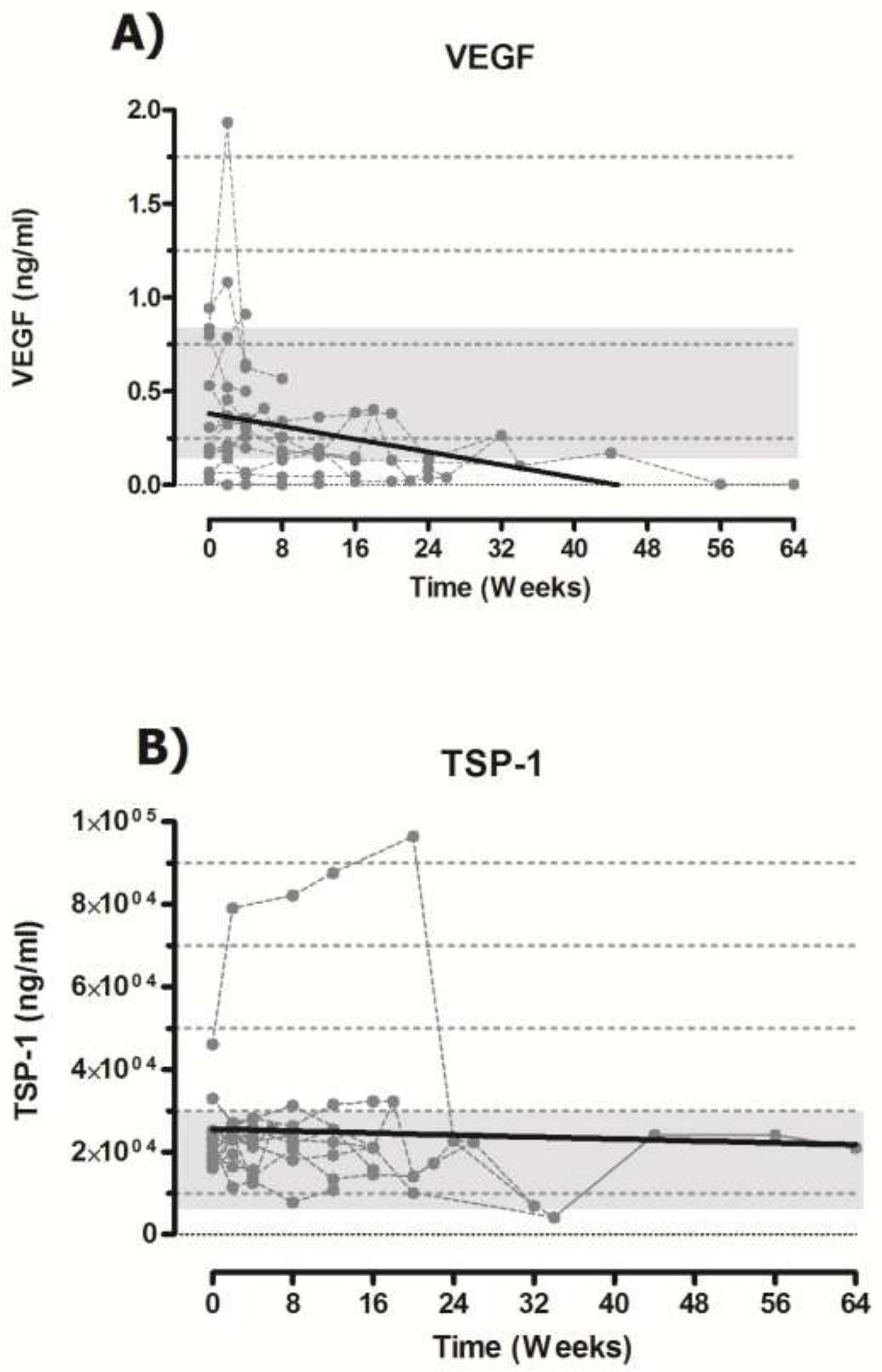
Online Resource 1
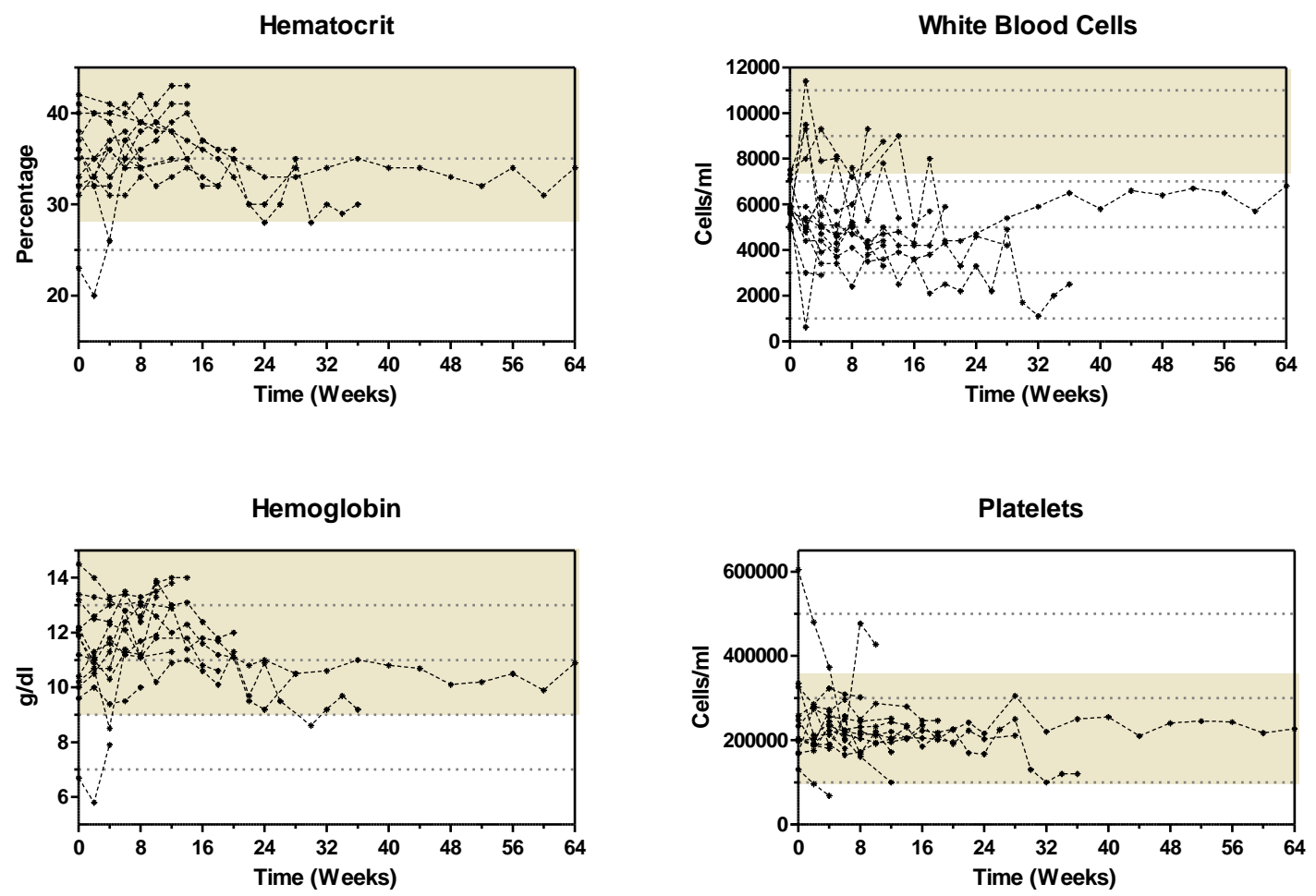

Online Resource 1. Evolution of hematologic parameters. The grey zones indicate the range of normal values. 
Online Resource 2
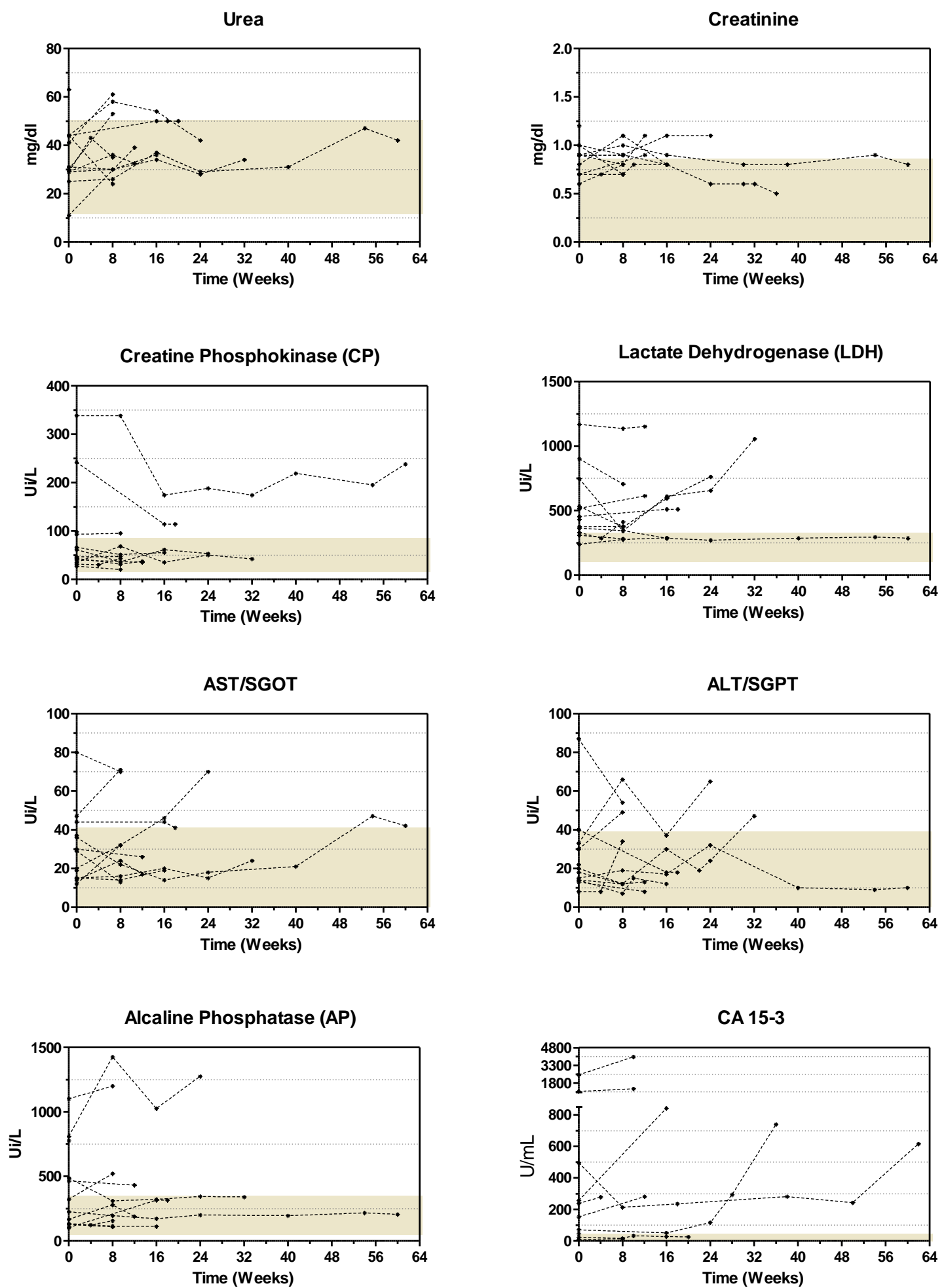

Online Resource 2. Evolution of serologic parameters. The grey zones indicate the range of normal values. Deviations from normality in values of the hepatic enzymes LDH and AP were caused by baseline disease. High levels of $\mathrm{CP}$ were present before the start of the treatment, and were not associated to the therapy. CA 15-3 antigen increased at the time of progression. 
Online Resource 3

\begin{tabular}{|c|c|c|c|c|c|c|c|c|c|c|c|c|c|c|c|c|}
\hline \multicolumn{17}{|c|}{ Quality of life during the follow-up, evaluated by the FACT-B questionnaire. } \\
\hline \multirow[b]{2}{*}{ Patient } & \multicolumn{5}{|c|}{ GP } & \multicolumn{5}{|c|}{ GS } & \multicolumn{5}{|c|}{ GF } & \\
\hline & BL & MT & ET & $\begin{array}{c}\boldsymbol{P} \\
(\boldsymbol{D R})^{+}\end{array}$ & $\boldsymbol{P}(\boldsymbol{D T})^{*}$ & BL & MT & ET & $\begin{array}{c}P \\
(D R)\end{array}$ & $P(D T)$ & BL & MT & ET & $\begin{array}{c}P \\
(D R)\end{array}$ & $P(D T)$ & BL \\
\hline 1 & 5 & 10 & 22 & \multirow{15}{*}{0.271} & \multirow{15}{*}{0.651} & 5 & 4 & 0 & \multirow{15}{*}{0.765} & \multirow{15}{*}{0.622} & 22 & 19 & 22 & \multirow{15}{*}{0.247} & \multirow{15}{*}{0.055} & 13 \\
\hline 2 & 4 & MS & 7 & & & 3 & MS & 4 & & & 4 & MS & 7 & & & 10 \\
\hline 3 & 3 & 5 & 7 & & & 10 & 9 & 11 & & & 2 & 4 & 3 & & & 1 \\
\hline 4 & 6 & 11 & 11 & & & 3 & 4 & 4 & & & 4 & 14 & 5 & & & 2 \\
\hline 5 & 6 & 2 & 1 & & & 5 & 6 & 4 & & & 16 & 8 & 8 & & & 10 \\
\hline 6 & 7 & 3 & 11 & & & 5 & 0 & 8 & & & 13 & 8 & 15 & & & 11 \\
\hline 7 & 5 & 13 & 4 & & & 12 & 4 & 3 & & & 3 & 3 & 5 & & & 11 \\
\hline 8 & 15 & 8 & 12 & & & 3 & 3 & 7 & & & 4 & 4 & 5 & & & 8 \\
\hline 9 & 15 & MS & 11 & & & 7 & MS & 0 & & & 18 & MS & 13 & & & 20 \\
\hline 10 & 28 & 15 & 24 & & & 5 & 17 & 13 & & & 20 & 16 & 17 & & & 28 \\
\hline 11 & 8 & 10 & 10 & & & 5 & 9 & 6 & & & 7 & 14 & 16 & & & 14 \\
\hline 12 & 8 & 8 & 28 & & & 4 & 4 & 1 & & & 9 & 6 & 17 & & & 6 \\
\hline 13 & 5 & 1 & 7 & & & 0 & 0 & 0 & & & 4 & 1 & 16 & & & 3 \\
\hline 14 & 25 & 5 & 1 & & & 0 & 0 & 0 & & & 20 & 7 & 7 & & & 8 \\
\hline 15 & 28 & 14 & 5 & & & 0 & 3 & 0 & & & 7 & 6 & 5 & & & 19 \\
\hline
\end{tabular}

GP: Physical well-being; GS: Social/family well-being; GF: Functional well-being; GE: Emotional wellbeing; AC: Additional concerns.

BL: Baseline; MT: Middle of treatment; ET: End of treatment.

$\mathrm{MS}=$ Missing data.

${ }^{+} \mathrm{P}(\mathrm{DR})=\mathrm{P}$ value $($ Wilcoxon signed-rank test $)$ during response $(\mathrm{BL}-\mathrm{MT}) ;{ }^{*} \mathrm{P}(\mathrm{DT})=\mathrm{P}$ value $($ Friedman

Test) during all treatment (BL-MT-ET). 
Online Resource 4

\begin{tabular}{|c|c|c|c|c|c|c|c|c|c|c|c|c|c|c|c|c|}
\hline \multirow[b]{2}{*}{ Patient } & \multicolumn{16}{|c|}{ ECOG Scale } \\
\hline & $\mathbf{0}$ & 4 & 8 & 12 & 16 & 20 & 24 & 28 & 32 & 36 & 40 & 44 & 48 & 52 & 56 & 60 \\
\hline 1 & 2 & 2 & 2 & 3 & & & & & & & & & & & & \\
\hline 2 & 2 & 2 & & & & & & & & & & & & & & \\
\hline 3 & 3 & 3 & 2 & & & & & & & & & & & & & \\
\hline 4 & 3 & 3 & 2 & 2 & & & & & & & & & & & & \\
\hline 5 & 2 & 1 & 1 & 2 & & & & & & & & & & & & \\
\hline 6 & 2 & 2 & 1 & & 1 & 1 & 1 & 1 & 1 & & & 1 & 1 & 1 & 1 & 1 \\
\hline 7 & 3 & 3 & 2 & 2 & 2 & 3 & & & & & & & & & & \\
\hline 8 & 1 & 1 & 2 & & & 1 & 2 & 2 & 2 & 1 & & & & & & \\
\hline 9 & 2 & 3 & & & & & & & & & & & & & & \\
\hline 10 & 1 & 1 & 2 & 3 & & & & & & & & & & & & \\
\hline 11 & 1 & 1 & 1 & 1 & 2 & 3 & & & & & & & & & & \\
\hline 12 & 1 & 1 & 1 & 1 & 1 & 1 & & & 2 & 3 & & & & & & \\
\hline 13 & 2 & 1 & 1 & 1 & 2 & 2 & 2 & & & & & & & & & \\
\hline 14 & 2 & 3 & & & & & & & & & & & & & & \\
\hline 15 & 2 & 1 & 2 & 1 & 1 & & & & & & & & & & & \\
\hline
\end{tabular}

Online Resource 4.Performance Status - ECOG Scale 